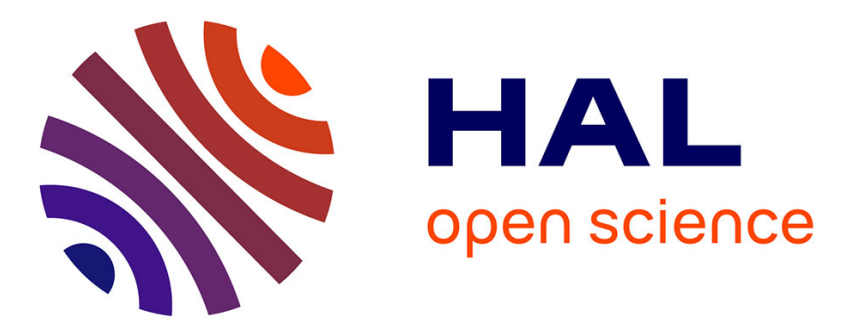

\title{
Mécanismes de co-construction et parcours identitaires : l'émergence de la parole dans le discours des usagers toxicomanes
}

\author{
Katja Ploog, Séverine Equoy Hutin, Sophie Mariani-Rousset, Sabrina \\ Hezlaoui-Hamelin, Laëtitia Grosjean, Line Pedersen
}

\section{To cite this version:}

Katja Ploog, Séverine Equoy Hutin, Sophie Mariani-Rousset, Sabrina Hezlaoui-Hamelin, Laëtitia Grosjean, et al.. Mécanismes de co-construction et parcours identitaires: l'émergence de la parole dans le discours des usagers toxicomanes. 2015. halshs-01385535

\section{HAL Id: halshs-01385535 \\ https://shs.hal.science/halshs-01385535}

Preprint submitted on 3 Nov 2016

HAL is a multi-disciplinary open access archive for the deposit and dissemination of scientific research documents, whether they are published or not. The documents may come from teaching and research institutions in France or abroad, or from public or private research centers.
L'archive ouverte pluridisciplinaire HAL, est destinée au dépôt et à la diffusion de documents scientifiques de niveau recherche, publiés ou non, émanant des établissements d'enseignement et de recherche français ou étrangers, des laboratoires publics ou privés. 


\title{
Mécanismes de co-construction et parcours identitaires : l'émergence de la parole dans le discours des usagers toxicomanes
}

\author{
Katja Ploog \\ LLL (UMR 7270 CNRS), Université d'Orléans \\ Séverine Équoy-Hutin \\ Sophie Mariani-Rousset \\ Sabrina Hezlaoui-Hamelin \\ ELLIADD, Université de Franche-Comté \\ Laetitia Grosjean \\ Université de la Réunion et ELLIADD \\ Line Pedersen \\ Université de Fribourg et LASA
}

Le projet "Parole émergée », initié en 2012 à l'Université de FrancheComté, est une recherche-action visant à saisir (documenter, décrire, analyser) le processus de construction identitaire qui se déroule au fil des interactions de soin en structure pluridisciplinaire. La relation soignantsoigné y est particulièrement riche en ce que ces structures proposent des parcours de soin à plusieurs voix, où l'usager rencontre plusieurs acteurs professionnels sur une durée qui peut être relativement longue et à intervalles très variables.

Nous faisons l'hypothèse que le processus de soin travaille l'identité discursive de l'usager addict et que l'usager engagé dans ce processus opère un travail identitaire au fil des interactions avec les soignants et que 
le professionnel joue un rôle fondamental dans ce processus de coconstruction. Notre objectif est d'observer cette mobilité identitaire de l'usager-addict dans le parcours de soin dans une perspective pluridisciplinaire, sous l'angle des interactions soignant-soigné et des traces écrites qui circulent au sein de la structure (le dossier de soin en particulier).

Après une présentation de notre problématique de recherche autour de l'identité discursive (section 1), nous détaillerons les enjeux interactionnels et communicationnels qu'implique le suivi en structure pluridisciplinaire, telle que l'Escale, contexte de l'étude (section 2). La section 3 sera destinée à expliciter les choix méthodologiques retenus. Le recueil des données étant encore en cours au moment où nous rédigeons ces lignes, nous nous contenterons de proposer dans la section 4 quelques pistes de réflexions que la conduite du projet permettra de développer.

\section{La (re-)présentation de soi dans le discours}

Le projet "Parole émergée " se construit avec une approche pluridisciplinaire: de ce fait, le concept d'identité qui a été travaillé dans nos disciplines respectives (sciences du langage, Sciences de l'InformationCommunication, Psychologie et Sociologie) doit faire l'objet d'un croisement disciplinaire nécessaire pour poser les bases du projet et travailler ensemble: dans le champ de l'analyse du discours, notamment, les travaux d'Amossy (2010) sur l'identité discursive éclairent sur le fonctionnement, les fonctions et les enjeux de la présentation de soi dans sa dimension intersubjective. Processus dynamique et social, à la fois maîtrisé et non maîtrisé, volontaire et involontaire, l'identité est plurielle et se négocie sur fond de représentations culturelles, collectives et doxiques. Dans ce projet, il nous faudra distinguer la présentation de soi telle qu'elle pourra être perçue à l'écoute des interactions enregistrées et 
In : Pascaline Lefort (ed.), Identité(s) : construction(s) et (re)construction(s) dans le discours. Ed. du Septentrion.

l'expertise de cette présentation de soi délivrée par les professionnels dans leur prise d'écriture (dossiers de soin).

\section{Autour de l'identité discursive}

Ricœur (1985) considère que l'identité narrative est constitutive de l'émergence du sujet "qui apparaît simultanément comme lecteur et comme auteur de sa propre vie »(de Gaulejac, 2002). Le paradigme psycho-sociologique interactionniste apporte en ce sens des outils conceptuels et analytiques féconds pour l'étude des interactions et ses incidences sur la construction identitaire. Selon Goffman (1973), le moi l'instance par laquelle l'individu s'interprète - ne peut exister qu'au regard d'une interprétation de la situation et de ses conventions partagées. Il ne s'agit pas d'appréhender le moi comme une interprétation d'un ou plusieurs rôles que l'individu manipulerait, mais il faut s'efforcer de penser les situations dans lesquelles l'individuation (Simondon, 1989) a lieu, c'est-à-dire penser la manière dont le moi se construit au cours de diverses interactions. Le moi est selon Gardella (2003) multiple en ce sens que la variété de situations dans lesquelles les normes, mêmes impérieuses, sont à respecter entraîne une variation d'actualisations du moi.

Dans ces variations, des détournements sont possibles au profit d'un rôle étranger à celui qui peut être imposé par les institutions (comme celui de patient ou d'usager). Le moi n'est pas intégralement re-déterminé dans chaque situation, il est aussi doté d'une réflexivité que Goffman appelle le quant-à-soi : l'idée que nous nous faisons de nous-mêmes avant une situation donnée, construite à partir d'une série d'interactions antérieures. Ce quant-à-soi n'existe que tant que l'individu le met à l'épreuve en situation. Pour Mead (1934), l’identité est représentée par le soi, qui se compose à la fois du moi (le conformisme, le respect des normes sociales) et du je (les réactions et ajustements avec la communauté) : "Ce n'est pas moi qui me crée moi-même : j'adviens plutôt à moi-même » (Jung, 1971 : 281). L'identité sociale est «le plus sûr registre que nous puissions consulter pour nous assurer de la consistance et de la continuité du Moi» (Rosset, 1999). La modalisation de l'activité observable dans les interactions de soin par les réitérations des demandes ou des thématiques de l'échange verbal nous permettra de déterminer les différentes modalités d'engagement des acteurs dans l'expérience de l'interaction.

D'un point de vue communicationnel, une conception non déterministe et constructiviste admet la part institutionnelle des rapports interpersonnels, mais elle "situe leur fondement de 'norme objective' au niveau intersubjectif et empirique de l'échange discursif » (Burger, 1994 : 253). Burger propose ainsi le terme de profil identitaire pour définir les 
images identitaires réciproques des sujets qui participent à la construction du « cadrage relationnel » (Burger, 1994 : 254). L'identité n'est jamais achevée, elle est " construite dans la dialectique de la reconnaissance intersubjective [...] (re)définie dialogiquement dans l'espace de l'interlocution »(Burger, $1994: 250)$. En ce sens, l'identité est un produit social en relation avec la reconnaissance communicationnelle qu'elle permet, au sein d'espace et d'enjeu particuliers (Ghiglione \& Trognon, 1993) ; mais elle est aussi un produit cognitif, puisque les normes se constituent à partir des représentations mentales que les sujets élaborent (Ghiglione, 1990). Ainsi, l'identité et le social peuvent être en partie accessibles en observant les activités discursives des sujets (Roulet, 1997). L'identité discursive est construite par le sujet parlant, tandis que l'identité sociale passe par la nécessité d'être reconnu par les autres (Charaudeau, 2009). Chabrol (2006) retrace la relation dynamique entre les identités psychosociales et les identités discursives, montrant que celles-ci relèvent de processus impliquant notamment des activités de remémoration narrative autobiographique (Ricœur, 1985).

Sur le plan linguistique, ces activités sont relayées par une compétence de différentes modalités langagières plus ou moins routinières (i.e. la représentation mémorielle des structures), de la connaissance la plus globale des normes sociales relatives aux interactions, par des macrostructures textuelles et des outils structurels permettant la gestion locale de la séquentialité (contextualization cues: Gumperz, 1982) aux constructions formulaires "figées ». Nous poserons que la validation - la reconduction/l'enracinement cognitif (Langacker, 1987 : entrenchment) vs la modification (Ploog, 2012 : élaboration) - de ces routines est liée, au moins en partie, à l'efficacité et la satisfaction relatives à l'action discursive.

Nous retiendrons de ces différents éclairages du concept les éléments suivants :

- D’une part, l'identité est alimentée par des événements auxquels participe le sujet, provoquée par lui ou non ; certains de ces événements sont mis en discours par le sujet qui les rapporte ou les évoque au cours des interactions. L'identité discursive est alors l'une des dimensions de l'identité, indissociablement liée à d'autres dimensions socio-culturelles qui structurent la vie de l'individu.

- D'autre part, l'identité discursive possède une dimension globale et permanente, en ce sens qu'elle est composée de l'ensemble des représentations forgées au fil des expériences de l'individu (sujet) et qui lui permettent d'organiser son agir discursif (e.g. Si je me 
In : Pascaline Lefort (ed.), Identité(s) : construction(s) et (re)construction(s) dans le discours.

Ed. du Septentrion.

rappelle bien) (Plazaola Giger \& Friedrich, 2005 : 247). Elle possède une autre dimension, dynamique, évolutive, en ce que le réinvestissement dans l'agir discursif conduit à la révision (reconduction ou modification) de ces représentations. Ainsi, l'identité discursive est soumise à un processus permanent de reconstruction.

Notre recherche vise à problématiser l'identité discursive sous trois aspects :

1. sa manifestation générale et fragmentaire dans la situation de soin,

2. les traces formelles de la reconstruction identitaire dans les interactions de soin,

3. son expertise/consignation par les professionnels dans le dossier de soin.

\section{L'émergence de l'identité discursive dans le parcours de soins}

L'appropriation de l'espace social - que ce soit par le biais de démarches institutionnelles ou les relations de la vie courante - repose sur l'identité sociale du sujet. Si la consommation de substances psychoactives entraîne une altération du sujet et conduit l'individu à modifier ses comportements, nous partons de l'hypothèse que des traces peuvent en être observées. Le cadre pluridisciplinaire du projet conduit de ce fait à formuler l'hypothèse d'un partage des observables. Les corrélations possibles sont diverses : cette altération peut constituer un handicap psychocomportemental qui pénalise le sujet dans son quotidien ; à l'inverse, certaines caractéristiques de la personnalité semblent prédestiner les sujets à l'abus de substances psycho-actives. Quoi qu'il en soit, la décision de faire appel à un professionnel pour prendre en charge sa toxicomanie est une étape importante - si ce n'est la première - d'un processus d'intégration/réintégration sociale.

L'élaboration de l'identité au fil des échanges constitue l'objet du présent projet. Cette question a été traitée dans la littérature du point de vue médical, psycho-social ${ }^{1}$ et socio-anthropologique ${ }^{2}$; en revanche, les approches linguistiques ont encore peu été utilisées, hormis dans le cadre de

${ }^{1}$ Anzieu et al, 1987 ; Bachmann \& Coppel, 1989; Bergeret, 1996 ; Hachet, 2002 ; Pedinielli, Rouan \& Bretagne, 2000 ; Taïeb, 2011 ; Valéa, 2005 ; Varescon, 2005.

2 Belin, 1996 ; Bourgois, 1995 ; Caiata Zufferey, 2006 ; Carricaburu \& Menoret, 2004 ; Delrieu, 1988 ; Ehrenberg, 1991 ; Fainzang, 1996 ; Gonet, 1992 ; Jamoulle, 2000 ; Jauffret-Roustide, 2004 ; Kangelari, 2003 ; Le Breton, 1992 ; Maquet, 1992 ; Niewiadomski, 2000 ; Pedersen, 2011. 
la problématique plus générale de l'interaction médecin-patient ${ }^{3}$. Cette interaction est un processus «par lequel les acteurs sociaux se constituent comme sujets » et construisent "leur identité par des jeux complexes de rôles et d'attentes réciproques" (Charaudeau, 2002) ${ }^{4}$.

Nous posons que l'usager construit et élabore son identité sociale spécifique, circonstanciée, dans le cadre de la relation de soin, au fil des échanges avec les professionnels du soin. Dès lors, quels sont les contenus clés autour desquels l'élaboration de cette identité est amorcée ? Quelles sont les étapes de cette élaboration et sont-elles généralisables? Quels sont les événements discursifs (Guilhaumou, 2006) qui renforcent ou freinent la dynamique de construction?

Nous posons en outre que les professionnels participent activement à la construction identitaire de l'usager par un accompagnement ciblé et différencié : quels moyens langagiers les professionnels utilisent-ils alors, lors des situations d'échange, pour soutenir l'usager dans ce processus de construction identitaire? Quels supports et quels types d'échanges les professionnels mettent-ils en œuvre pour garantir la cohésion de cette approche fragmentaire?

Cette première approximation fait comprendre le fait identitaire comme processus, inscrit dans le temps. En conséquence, les opérations qui seront conduites pour vérifier ces hypothèses feront appel à une approche dynamique de la communication, offrant une observation apte à varier les différentes échelles temporelles :

- au niveau micro, la séquentialité de l'interaction interroge la dynamique qui génère l'étayage de l'identité discursive en termes de mobilité langagière: l'élaboration discursive sera observée dans ses caractéristiques prosodiques et syntaxiques ;

- au niveau méso, la gestion des rapports de places entre les interlocuteurs sera observée par la gestion des tours de parole en général et par les ressorts utilisés par le professionnel et par l'usager-locuteur pour construire la cohérence entre les fragments livrés au fil du parcours ;

- au niveau des macromodalités de circulation du discours, l'on observera la dispersion identitaire au travers de l'intervention de différents acteurs.

${ }^{3}$ Furchner \& Gülich, 2002 / Gülich, 2005, 2012 ; Péréa, Levivier \& Belz Ceria, 2013 ; De Fornel \& Verdier, 2014

${ }^{4}$ L'entrée « Locuteur » (Charaudeau, $2002: 350-351$, cité par Chabrol 2006). 
In : Pascaline Lefort (ed.), Identité(s) : construction(s) et (re)construction(s) dans le discours. Ed. du Septentrion.

\section{Les axes de recherche}

L'exploitation des données se décline en trois axes thématiques : identité et altérité dans l'interaction; émergence du sujet et élaboration formelle du discours; et le sujet dans la circulation des écrits professionnels.

\section{Identité et altérité dans l'interaction}

L'identité relève d'un entre-deux : semblable et dissemblable, singularité et altérité, individu et collectif, unité et différenciation, objectivité et subjectivité (de Gaulejac, 2002). Cette émergence de l'identité découle d'une rencontre entre deux altérités, permettant l'accès aux interactions (Goffman, 1973 ; 1974). L'estime de soi se construit en fonction de la possible évolution de la parole, libre de formuler la réalité vécue par l'individu. Cette structuration de l'identité se fait dans le temps et au cours de toute la vie. Le professionnel, par ses interventions, participe à l'émergence d'une parole dans laquelle l'identité de l'usager se construit. La prise en charge par la structure accompagne le sujet dans la construction de ses limites, de sa place et de son identité (Anzieu et al., 1987). L'usager, parfois contraint juridiquement de venir dans le lieu, ne le perçoit pas forcément. C'est donc l'échange, l'empathie, le cadre, la prise en charge par la structure et les propositions qui lui sont faites, qui permettent de rétablir une meilleure image de soi au fil du temps, via la parole. Autrui, source de difficultés, est également celui par qui le sujet se construit. "Plus la conscience de l'autre est forte, plus fortement se construit la conscience identitaire de soi » (Charaudeau, 2009:16).

Dans les discours recueillis au cours des entrevues entre acteurs professionnels et usagers, nous chercherons la part de la parole émergente qui «échappe » et amène à la construction de soi, à l'individuation, à sa présence effective au monde des humains (Kerbrat-Orecchioni, 2005; Simondon, 1989; Van Hooland, 2002). À partir des éléments du parcours de vie avant la prise en charge du sujet, nous étudierons l'évolution du processus en fonction du suivi d'un même sujet, au cours des différents échanges.

\section{Émergence du sujet et élaboration formelle du discours}

Si le discours construit la relation à l'altérité - et le Soi -, l'identité sociale doit être raisonnée en corrélation avec la mobilité discursive, où le dépassement des obstacles communicatifs représente le dépassement de soi. Cette identité discursive dynamique sera envisagée sur les plans de l'émergence syntaxique et de l'architecture sonore. Les identités des actants/acteurs sociaux dépendent «des statuts sociaux et des rôles langagiers auxquels ceux-ci donnent accès » (Fillietaz, $2004: 16$ ). 
La modalité parlée fait apparaître nombre d'éléments qui semblent parasiter la progression du discours: disfluences, piétinements syntaxiques, ambiguités structurelles. Construire un discours consiste à élaborer la densité d'une identité en deux dimensions, l'une temporelle, l'autre structurelle. L'objectif de cet axe consiste à mettre en lumière les mécanismes par lesquels les locuteurs parviennent à résoudre les tensions entre linéarité et structure, condition nécessaire pour l'élaboration du discours en temps réel. Les enjeux majeurs en sont la cohésion, et la cohérence globale de la présentation de soi dans le discours. Dans une perspective théorique inspirée des Grammaires des Constructions (Hopper, 2004 ; Beckner et al., 2009) et de l'analyse conversationnelle (Gülich \& Mondada, 2008), nous nous intéresserons à l'élaboration syntaxique du discours sur trois échelles temporelles :

- l'émergence constructionnelle en temps réel, dans le déroulement de l'entretien entre soignant et usager ;

- le développement de la mobilité discursive du locuteur, en particulier par la mise en évidence des procédés syntaxiques ;

- sur un plan plus global, le relevé longitudinal permettra d'esquisser l'émergence linguistique par une remise en perspective des données en termes de stabilité ( $v s$ variabilité / évolution).

\section{Le sujet dans la circulation des écrits professionnels}

L'identité circonstanciée de l'usager se construit et évolue dans les traces que celui-ci laisse dans la structure par l'intermédiaire des mots du professionnel. Il s'agit d'observer l'évolution de la construction identitaire et de la figure de l'usager construite par le professionnel dans la perspective d'une analyse de discours en lien avec une demande sociale (Cislaru, Pugnières-Saavedra \& Sitri, 2010) et plus spécifiquement dans la perspective d'une analyse de la présentation de soi dans le discours (Amossy, 2010) : l'objectif est d'observer, dans les écrits circulants au sein de la structure, l'évolution de l'identité de l'usager telle qu'elle est perçue par les professionnels et son conditionnement par les modalités de sa prise en charge langagière par les acteurs professionnels. En d'autres termes, il s'agit de penser la co-construction identitaire en considérant la prise d'écriture dans sa dimension agissante. La recherche est donc doublement orientée: vers la construction d'un "diagnostic identitaire " (identité expertisée) de l'usager, d'une part, et vers les pratiques professionnelles (Borzeix \& Fraenkel, 2005), d'autre part; on analyse donc la prise en charge langagière de l'usager à travers les traces écrites produites par les professionnels et la dispersion identitaire, tel un puzzle, que l'intervention de différents acteurs suscite. 
In : Pascaline Lefort (ed.), Identité(s) : construction(s) et (re)construction(s) dans le discours.

Ed. du Septentrion.

Ainsi, l'analyse porte notamment sur l'évolution, au fil des documents, de l'identité de l'usager à travers la perception de cette identité par le professionnel (Laé, 2008) : quels sont les axes identitaires exploités dans le cadre du suivi par les professionnels de la structure? Les modalités par lesquelles cette identité est prise en charge, schématisée (Grize, 1990) par un point de vue professionnel relativement aux objectifs d'accompagnement de la structure, paraissent fondamentales : en d'autres termes, comment l'identité de l'usager se transforme-t-elle en "identité expertisée » ? (Léglise \& Garric, 2012).

\section{Le contexte de l'étude : l'accueil de l'usager en structure pluridisciplinaire}

L'enquête est menée en collaboration avec la structure d'accueil l'Escale, un centre de soins, d'accompagnement et de prévention en addictologie (CSAPA) à Vesoul en Haute-Saône. L'Escale a pour mission d'assurer une prise en charge des addictions, en répondant par des soins psychologiques, médicaux, infirmiers et un accompagnement socioéducatif de l'usager de produits psycho-actifs, dont notamment la mise en place de traitements de substitution (méthadone, subutex, etc.). L'accompagnement social, s'il n'est pas la priorité affichée de la structure, peut parfois comprendre une (ré)insertion professionnelle.

L'Escale héberge deux établissements médico-sociaux : d'un côté, l'une des quatre antennes du CSAPA de Haute-Saône, qui est une structure généraliste; de l'autre, un centre d'accueil et d'accompagnement à la réduction des risques pour usagers de drogues (CAARUD). Si ces deux structures visent en principe des missions et des publics d'usagers distincts, elles sont dans la réalité souvent confondues par les usagers sous le chapeau de l'Escale; par ailleurs, cette configuration semble offrir une 
plus grande souplesse pour les professionnels dans la prise en charge des difficultés du public.

L'équipe de l'Escale est constituée d'un assistant de direction (également éducateur), de cinq médecins (un seul rattaché à la structure, les autres intervenant sur des créneaux fixes mais très restreints, sinon principalement en libéral), deux éducateurs, deux infirmières, deux psychologues et une animatrice positionnée à l'accueil.

En 2010, l'Escale a accueilli environ 800 usagers, la moitié d'entre eux pour la première fois. Le public se compose pour la plupart d'usagers d'alcool et d'héroïne, avec, dans de nombreux cas, des poly-addictions; devant la diversité des profils, les incertitudes sur les pratiques réelles (parfois très chaotiques) des usagers, il n'est pas envisagé pour l'heure de procéder à une typisation plus précise du public dans le cadre de l'étude. La moyenne d'âge pour les deux types d'addictions déclarées est de 27 ans, avec une nette différence entre les différentes addictions : plus de 40 ans pour l'alcool et moins de 30 ans pour l'héroïne. Selon leur propre déclaration, le premier contact à l'Escale des usagers d'opiacés a lieu environ un à deux ans après les premières expériences - à un moment, donc, où la consommation du produit est déjà fortement installée.

L'usager s'adresse à l'Escale le plus souvent pour solliciter la mise en place d'un traitement de substitution. Au fil des contacts entre usager et soignants, d'autres problèmes (d'ordre social, psychologique, psychiatrique, somatique, etc.) surgissent fréquemment qui nécessitent une prise en charge spécifique, parfois même avant de pouvoir envisager un traitement substitutif.

L'objectif de l'Escale est de stabiliser suffisamment la personne pour ensuite passer le relais au médecin traitant. Un parcours se dessine alors, en fonction de l'orientation à donner.

\section{Le parcours de soin en CSAPA}



Ed. du Septentrion.

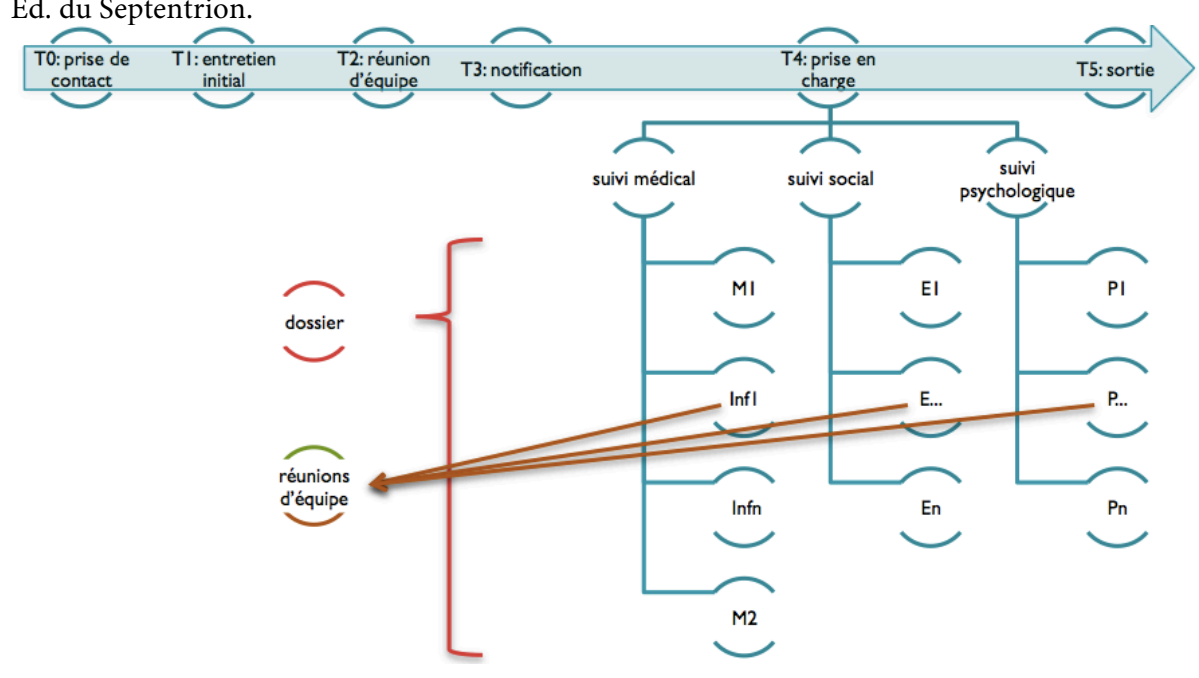

Fig. Parcours de prise en charge

Lorsqu'un usager contacte l'Escale pour la première fois, l'animatrice de l'accueil enregistre sa demande et lui propose un rendez-vous avec un interlocuteur adapté - le plus souvent un éducateur. En effet, les droits sociaux (sécurité sociale, logement, sécurité financière, etc.) sont à la base d'un suivi médical efficace. L'éducateur détaille la demande et établit le dossier "administratif » de la personne, puis en rend compte à l'équipe. Cette dernière convient de la prise en charge qui peut être proposée à l'usager, conformément ou non à la demande. Celle-ci peut consister en un traitement de substitution, mis en place par le médecin puis suivi au quotidien par une infirmière de la structure; ou bien, parfois, et parce que l'instabilité notoire de l'usager ne permet pas (encore) un tel traitement, l'usager est réorienté sur l'éducateur qui doit l'accompagner pour résoudre d'autres problèmes (comme retrouver une couverture sociale) avant la mise en place d'une éventuelle mesure médicale. Au fil de la prise en charge, le soignant "détecte» les particularités et les fragilités de l'usager, susceptibles de faire l'objet d'une prise en charge complémentaire. Lorsque cela est jugé bénéfique, et à tout moment de la prise en charge, l'usager peut être (ré)orienté sur un autre interlocuteur : éducateur, médecin, psychologue, pour œuvrer en faveur d'une amélioration de sa situation. À noter que le refus d'une rencontre avec le psychologue n'entraîne pas de conséquence dans l'accueil du sujet et son traitement. Un certain nombre d'usagers est suivi à l'Escale depuis plusieurs années. C'est précisément cet aspect de l'action dans la durée qui nous a conduit à établir un projet de recherche en collaboration avec ce centre, dans la perspective d'un suivi longitudinal des sujets. 


\section{Objectifs}

L'objectif global consiste à décrire le processus interactionnel qui permet au locuteur de se (re)construire, ce qui implique de faire face à plusieurs défis :

- dégager les stratégies formelles investies dans l'élaboration discursive ;

- dégager les mécanismes de co-construction;

- identifier les parcours identitaires de chacun, la quête passant par la confrontation à l'autre ;

- considérer l'ensemble des supports qui portent les traces de ce processus et qui font la mémoire de l'institution.

La méthodologie de recueil des données a été conçue sur mesure, afin de faire face à la particularité du terrain (données sensibles, interlocuteurs multiples, la routine d'enregistrement) sans sacrifier le relevé rigoureux. En contrepartie, les objectifs ont dû être renégociés à la baisse.

\section{Corpus}

Le recueil de données a été contractualisé sur une durée de dix-huit mois. Cette durée relativement courte pour une étude longitudinale s'explique principalement par des contraintes structurelles (responsabilité, financement). Si l'expérience est concluante (pour l'équipe professionnelle y compris), une nouvelle période de recueil peut être projetée. Le recueil comporte notamment :

- l'enregistrement d'entretiens soignant-soigné : la transcription orthographique intégrale, idéalement aligné sur le signal sonore, permettra de repérer les éléments de contenu et de forme pertinents à l'analyse; il s'agira de problématiser par ailleurs les postures et les aspects relationnels de l'échange dissymétrique et formel ainsi que les techniques d'entretien libre et leurs grilles de lecture associées ;

- le recueil des traces écrites internes, précisément dans leur évolution : l'objectif consistera d'abord à identifier les différents genres professionnels (papiers, électroniques) qui circulent dans la structure, puis d'établir leurs fonctions dans la construction identitaire de l'usager du point de vue des professionnels;

- le recueil d'entretiens complémentaires avec les professionnels de la structure, concernant leurs pratiques d'échange avec et sur les usagers. Ces entretiens seront programmés à l'issue du recueil 
In : Pascaline Lefort (ed.), Identité(s) : construction(s) et (re)construction(s) dans le discours.

Ed. du Septentrion.

des données orales, pour éviter de perturber leur pratique habituelle ou d'induire des biais dus à des questionnements sur nos objets de recherche.

\section{Traitement des données}

Les données orales sont relevées par les professionnels eux-mêmes, qui recueillent les consentements des usagers et gèrent les enregistrements. Les différentes salles où sont dispensés les actes (cabinet médical, infirmerie, bureau d'éducateur/psychologue) sont équipées d'enregistreurs et micros, certes visibles mais très discrets, que les professionnels arment eux-mêmes en fonction de la personne reçue. Seul l'usager ayant donné son accord explicite est enregistré. Les professionnels ont pour consigne de déclencher l'enregistrement dès que l'usager entre dans la pièce et de l'arrêter à son départ. Selon la situation, de nombreuses variations mineures sont recensées: par exemple, comme les professionnels vont généralement chercher les usagers en salle d'attente, les enregistrements ne comprennent que rarement les salutations d'ouverture. Notons aussi que nous avons accordé aux professionnels la liberté de juger s'il faut interrompre un enregistrement en cours.

Les chercheurs récupèrent les données et les archivent par leur date et un code usager et professionnel. Une première écoute rapide permet de repérer les passages sensibles, qui font ensuite l'objet d'une anonymisation et offrent déjà des pistes de réflexion. Ces données brutes sont archivées en l'état dans l'attente d'une transcription éventuelle, la cinquantaine d'heures enregistrées nous obligeant à un choix cohérent de retranscription - notre principal critère étant, au-delà de la qualité du contenu, la possibilité d'entendre un usager dans la durée. 
Le recueil a été terminé en 2014, les transcriptions sont en cours; dans la suite, nous allons esquisser les pistes d'exploitation qui se dessinent.

\section{Résultats attendus}

La mise en perspective des données orales, par rapport aux écrits professionnels et aux entretiens oraux effectués avec les acteurs, permet d'observer l'articulation entre les éléments identitaires fournis par l'usager pendant l'entretien et les traces consignées au sein de la structure dans les différents écrits professionnels. L'étude vise ainsi à mettre en exergue l'articulation des différentes composantes (documents, stratégies, phases du processus) dans la communication professionnelle, et leur contribution à la relation de soin.

Au-delà des objectifs scientifiques "internes", détaillés supra, nous espérons pouvoir offrir à la structure qui a accepté d'être notre terrain de recherche un regard éclairé sur l'échange verbal, qui représente l'outil de travail central de ces professionnels dans leur relation avec les usagers. Notre étude pourra servir enfin à remettre en perspective l'outil d'évaluation interne stipulé par l'Agence Régionale de la Santé, qui préconise de «favorise[r] l'expression des usagers pour la mise en œuvre de leur accompagnement individuel $» .^{5}$

Dans son cadre sociétal, le bénéfice direct de cette étude est d'expliciter le rôle des acteurs professionnels et des CSAPA dans les parcours de soin des usagers de substances psycho-actives et dans le processus de leur (re)construction identitaire.

\section{Bilan intermédiaire}

Les enregistrements ont été effectués de janvier 2013 à février 2014 par les professionnels eux-mêmes, qui avaient à leur disposition un enregistreur. Le corpus est constitué désormais d'environ 100 heures enregistrées - bien trop pour faire l'objet d'une analyse systématique complète. Or, le bilan du recueil montre quelques faiblesses, qui infléchiront les choix d'exploitation du matériau : certains professionnels ont pu souligner la lourdeur de gestion des enregistrements dans le quotidien des rendezvous et avouent avoir été pris de court - tous persistent à souligner néanmoins l'intérêt de l'étude et ont donné leur avis favorable au préalable, après une phase de concertation de deux mois, pendant laquelle plusieurs rencontres entre chercheurs et professionnels ont été organisées; il nous a fallu admettre que le "puzzle » allait rester incomplet, que nous n'obtiendrions pas des journées entières documentées à l'infirmerie,

\footnotetext{
${ }^{5}$ Source : ANPAA70, Rapport annuel d'activité 2011.
} 
In : Pascaline Lefort (ed.), Identité(s) : construction(s) et (re)construction(s) dans le discours. Ed. du Septentrion.

ni d'enregistrements de locuteurs dont toutes les venues à l'Escale auraient été consignées.

En vue des analyses, nous avons fait le choix de focaliser notre attention sur certains locuteurs, qui répondent non pas à des critères démographiques ou psycho-sociaux, mais dont les données rendent compte au mieux de nos priorités d'analyse : sont transcrites les données d'usagers réguliers ou faisant appel à la structure de façon répétée, qui fréquentent régulièrement l'Escale et y rencontrent différents interlocuteurs professionnels. Un autre critère est le suivi d'un usager depuis le début de sa prise en charge (hormis l'entretien préliminaire, qui a été exclu du relevé pour ne pas mettre en péril la relation de soin avant même sa stabilisation).

Sur le plan des écrits professionnels, une première observation ciblée de plusieurs dossiers d'usagers conservés dans la structure semble confirmer nos hypothèses initiales: on constate en effet un tissage particulièrement étroit ainsi qu'une complémentarité significative des points de vue des acteurs professionnels (éducateurs, médecins, psychologues). Au-delà du partage d'informations et d'une vision commune de l'usager à laquelle les réunions hebdomadaires entre les professionnels peuvent concourir, chaque professionnel contribue, de son point de vue c'est-à-dire depuis son «champ d'action professionnelle» à écrire l'identité globale de l'usager dans l'addiction. C'est ainsi la figure de l'usager (c'est-à-dire, la représentation que s'en font les professionnels) dans ses différentes formes de dépendances, en lien avec une situation sociale le plus souvent fragile, qui vient s'altérer au fil des prises d'écriture. Cette figure tend de fait à s'écrire dans un rapport à une "norme » sociale implicite, intégrée par le professionnel, et vers laquelle il s'agit d'orienter le sujet. Cette normalisation des usagers est visée en référence à des normes capacitaires (Laforgue, 2009) telles que travailler, se déplacer, être stable, avoir des relations sociales, avoir des projets, etc.).

Les premières explorations des données orales recueillies corroborent l'idée qu'une structure pluridisciplinaire permet l'émergence de modalités de communication spécifiques: en effet, de nombreux cas de transgression du «contrat de communication » (Couty, 2011) ont été observés, où un usager aborde par exemple des questions médicales avec l'éducateur ou des problèmes économiques avec l'infirmière (Équoy-Hutin \& Mariani-Rousset, 2016). Reste à établir plus précisément comment ces transgressions s'insèrent dans l'interaction en cours et comment elles sont prises en considération par le personnel soignant à court et à moyen terme. 


\section{Bilan}

L'objectif de ce projet, dont les analyses sont à peine amorcées au moment de cette rédaction (cf. p.ex. Ploog 2014), est de problématiser quelques aspects dynamiques de l'identité discursive dans le cadre d'un processus de soin, et ce par une approche pluridisciplinaire voire interdisciplinaire si des zones de recouvrement se font jour.

Les chercheures associées autour de ce projet sont issus de traditions disciplinaires différentes et variées. Plutôt que d'y voir un obstacle à la cohérence des analyses, nous considérons ce fait comme une opportunité pour proposer une approche intégrée du discours, alliant analyse linguistique (structurelle) et analyse thématique, puis pour poser des questionnements complémentaires sur la construction du sujet, l'usager toxicomane en l'occurrence. La méthodologie de recherche elle-même est expérimentale, en ce que nous cherchons à développer une approche fragmentaire de l'identité.

Notons aussi que l'angle de vue adopté se veut novateur: la plupart des études sur les addictions et les échanges soignant-soigné ont été menées dans d'autres disciplines que les sciences du langage telles que la sociologie, la psychologie, la psychiatrie, la médecine, et dans d'autres visées. Ainsi, la construction d'une problématique autour du langage, qui place celui-ci comme objet même de l'investigation (au lieu d'envisager le langage comme transmetteur d'un objet tiers) apportera à n'en pas douter de nouveaux éclairages sur le fonctionnement du soin en addictologie, et sur le fonctionnement des soins dispensés par la parole en général.

Enfin, la saisie unique du discours comme une construction collaborative permet en outre de dépasser le clivage usager/profane $v s$ professionnel, en projetant le sens en général, et l'identité discursive en particulier, comme un ensemble co-construit, hétérogène, composite et dynamique.

\section{Bibliographie}

Amossy R., La présentation de soi : ethos et identité verbale, Paris, PUF, 2010.

Anzieu D. et al., Les enveloppes psychiques, Paris, Dunod, 1987.

Bachmann Ch. \& Coppel A., Le dragon domestique, Paris, Albin Michel, 1989.

Beckner C. \& Bybee J., "A Usage-Based Account of Constituency and Reanalysis ", in Ellis N.-C. \& Larsen-Freeman D. (eds.): Language as a complex adaptive system, Language Learning, Chichester (West Sussex, UK) / Malden (Massachussetts, USA), Wiley-Blackwell, 2009, nº 59 (suppl. 1), p. 27-46.

Belin M., Laisse-moi être : libres paroles de toxicomanes, éd. Interdépendances, 1996.

Bergeret J., Toxicomanie et personnalité, Paris, PUF, 1996. 
In : Pascaline Lefort (ed.), Identité(s) : construction(s) et (re)construction(s) dans le discours. Ed. du Septentrion.

Borzeix A. \& Fraenkel B. (éds.), Langage et travail : communication, cognition, action, Paris, CNRS éditions, 2005 [2001].

Bourgois Ph., En quête de respect. Le crack à New York, Paris, Seuil, 2001 [1995].

Burger M., « (Dé)construction de l'identité dans l'interaction verbale : aspects de la réussite énonciative de l'identité ", Cahiers de linguistique française, 1994, $\mathrm{n}^{\circ} 15$, p. $235-264$.

Caiata Zufferey M., De la toxicomanie à la conventionalité, Sociologie des sorties de la drogue à l'époque de la réduction des risques, Zürich, Éditions Seismo, 2006.

Carricaburu D. \& Menoret M., La sociologie de la santé, Paris, Armand Colin, 2004.

Chabrol C., "Identités" sociales et discursives", Questions de communication, 2006, $n^{\circ} 9$, p. 5-27.

Charaudeau P., "Identité sociale et identité discursive. Un jeu de miroir fondateur de l'activité langagière " in Charaudeau P. (dir.), Identités sociales et discursives du sujet parlant, Paris, L'Harmattan, 2009, p. 16-28.

Charaudeau P., Maingueneau D. (éds), Dictionnaire d'analyse du discours, Paris, Seuil, 2002.

Cislaru G., Pugnière-Saavedra F. \& Sitri F. (coord.), «Analyse de discours et demande sociale : le cas des écrits de signalement ", Les Carnets du Cediscor, Paris, Presses Sorbonne Nouvelle, 2008.

Couty B., L'Usage, le Contrat et le Service, Bruxelles, Books on Demand, 2011.

Delrieu A., L'inconsistance de la toxicomanie : contribution à l'histoire des discours et des pratiques médicales, Paris, éd. Navarin, 1988.

Ehrenberg A. (dir.), Individus sous influence, drogues, alcool, médicaments psychotropes, Paris, Éditions Esprit, 1991.

Équoy-Hutin S., Mariani-Rousset S., "Sur les traces du secret : enquête sur la relation de soin et d'accompagnement de l'addiction ", ¿ Interrogations? $\mathrm{n}^{\circ} 22$ : "L'enquêteur face au secret", juin 2016.

Fainzang S., Ethnologie des anciens alcooliques. La liberté ou la mort, Paris, PUF, coll. ethnologies, 1996.

Filliettaz L., "Les modèles du discours face au concept d'action : présentation ", Cahiers de Linguistique Française, 2004, n 26, p. 9-23.

Fornel M. (de), Verdier M., Aux prises avec la douleur. Analyse conversationnelle des consultations d'analgésie, Paris, éd. de l'EHESS, Coll. "Cas de figure", 2014.

Gardella, É., « Du jeu à la convention. Le self comme interprétation chez Goffman », Tracés, 2003, $\mathrm{n}^{\circ} 4$, p. 21-42.

Gaulejac (de) V., "Identité » in Barus-Michel J. et ali. (dirs) : Vocabulaire de psychosociologie, références et positions, Paris, Érès, 2002.

Goffman E., La mise en scène de la vie quotidienne, tome 1 : la présentation de soi, Paris, Édition de Minuit, 1973

Goffman E., Les rites d'interaction, Paris, Édition de Minuit, 1974.

Gonet L., Adolescents, drogues et toxicomanie, éd. Lyon Chronique sociale, 1992.

Grize J.-Bl., Logique et Langage, Gap, Ophrys, 1990. 
Furchner I. \& Gülich E., "L'expertise des patients dans l'élaboration d'un diagnostic médical. Analyse linguistique d'entretiens avec des patients souffrant de crises épileptiques ou non-épileptiques», in Bulletin suisse de linguistique appliquée, 2002, $\mathrm{n}^{\circ} 74$, p. 83-107.

Ghiglione R., « Le qui et le comment ? » in Ghiglione R., Bonnet C. \& Richard J.F. (eds) : Traité de psychologie cognitive 3, Paris, Dunod, 1990.

Ghiglione R. \& Trognon A., Où va la pragmatique? De la pragmatique à la psychologie sociale, Grenoble, Presses Universitaires de Grenoble, 1993.

Guilhaumou J., Discours et événement. L'histoire langagière des concepts, Besançon, Presses univ. de Franche-Comté (Annales litt. de l'UFC, série « Linguistique et sémiotique », $\left.\mathrm{n}^{\circ} 47\right), 2006$.

Gülich E., «Unbeschreibbarkeit: Rhetorischer Topos - Gattungsmerkmal Formulierungsressource " in Gesprächsforschung, online Zeitschrift zur verbalen Interaktion, $2005, \mathrm{n}^{\circ} 6, \mathrm{p} .222-244$.

Gülich E., "Conversation analysis as a new approach to the differential diagnosis of epileptic and non-epileptic seizure disorders", in Egbert M. \& Deppermann A. (eds.): Hearing Aids communication. Integrating social interaction, audiology and user centered design to improve communication with hearing loss and hearing technologies, Mannheim, Verlag für Gesprächsforschung, 2012, p. 146-158.

Gülich E. \& Mondada L., Konversationsanalyse. Eine Einführung am Beispiel des Französischen, Tübingen, Niemeyer, 2008.

Gumperz J.-J., Discourse Strategies, Cambridge, Cambridge University Press, 1982.

Hachet P., Psychologue dans un service d'aide aux toxicomanes, Paris, Érès, 2002.

Hopper P.-J., "The Openness of Grammatical Constructions», Chicago Linguistic Society, 2004, n ${ }^{\circ}$ 40, p. 239-256.

Jamoulle P., Drogues de rue. Récits et styles de vie, Paris/Bruxelles, De Boeck Université, 2000.

Jauffret-Roustide M. (dir.), Les drogues, approche sociologique, économique et politique, Paris, Études de la Documentation Française, 2004.

Jung C. G., Les racines de la conscience, Paris, Buchet Chastel, 1971.

Kangelari M., Toxicomanie, science du langage, une approche clinique, Paris, L'Harmattan, 2003.

Kerbrat-Orecchioni, C., Les actes de langage dans le discours: théories et fonctionnement, Paris, Armand Colin, 2005.

Laé J.-Fr., Les nuits de la main courant : écritures au travail, Paris, Stock, 2008.

Laforgue D., « Pour une sociologie des institutions publiques contemporaines: Pluralité, hybridation et fragmentation du travail institutionnel », Socio-logos. Revue de l'association française de sociologie [En ligne], $\mathrm{n}^{\circ} 4$, 2009. URL: http://socio-logos.revues.org/2317

Langacker R., Foundations of cognitive grammar I, Stanford, Stanford University Press, 1987.

Le Breton D., Conduites à risques, Paris, PUF, 1992.

Léglise I. \& Garric N., Discours d'experts et d'expertise, Bern, Peter Lang, 2012. 
In : Pascaline Lefort (ed.), Identité(s) : construction(s) et (re)construction(s) dans le discours.

Ed. du Septentrion.

Levivier M. \& Perea Fr., Belz Ceria I. \& Collectif, Parole et addiction, Paris, Érès, 2013.

Maquet Cl., Toxicomanie et formes de la vie quotidienne, Bruxelles, Pierre Mardaga, 1992.

Mead G. H., Mind, Self and society, Chicago, University of Chicago Press, 1934.

Niewiadomski C., Histoire de vie et alcoolisme : à la recherche d'un espace de construction de sens avec les personnes alcooliques, Paris, éd. Seli Arslam, 2000.

Pedersen L.-K., Addictologie et savoirs profanes. Une approche sociologique, Besançon, Université de Franche-Comté (Mémoire de Master sous la dir. de D. Jacques-Jouvenot), 2011.

Pedinielli J.-L., Rouan G. \& Bretagne P., Psychopathologie des addictions, Paris, PUF, 2000.

Plazaola Giger I., \& Friedrich J., « Comment l'agent met-il son action en mots? Analyse d'entretiens auprès d'enseignants " in Filliettaz L., \& Bronckart J.-P. : L'analyse des actions et des discours en situation de travail. Concepts, méthodes et applications, Louvain-la-Neuve, Peeters, BCILL, 2005.

Ploog K., Approche syntaxique de la dynamique du discours oral. Application à quelques dynamiques urbaines dans le monde francophone et hispanique, Université de Nice-Sophia-Antipolis : HDR, 2012.

Ploog K., "Répétition lexicale et variation constructionnelle dans le discours spontané ", in Pop, Liana \& Curea Ana Maria (eds), L'oral: formes émergentes, corpus et modélisations, Studia Universitatis Babes-Bolyai. Seria Philologia n² 4 2014, p. 11-30.

Ricœur P., Temps et Récit III, Paris, Gallimard, 1985.

Rosset C., Loin de moi. Étude sur l'identité, Paris, éd de Minuit, 1999.

Roulet E., "L'analyse du dialogue comme forme et comme activité discursives », in Luzzati D. et al. (éds) : Le Dialogique, Berne, Lang, 1997, p. 97-122.

Simondon G., L'individuation psychique et collective, Paris, Aubier, 1989.

Taïeb O., Les histoires des toxicomanes. Récits et identités dans les addictions, Paris, PUF, 2011.

Valéa D., Toxicomanie et conduites addictives, Paris, éd. Heures de France, 2005.

Van Hooland M., La parole émergente. Approche psycho-sociolinguistique de la résilience. Parcours théorico-biographique, Paris, L'Harmattan, 2002.

Varescon I., Psychopathologie des conduites addictives : alcoolisme et toxicomanie, Paris, Belin, 2005. 